SUPPLEMENTARY MATERIAL

for the

full communication

entitled

\title{
Modular Total Synthesis of Lamellarin D
}

authored by

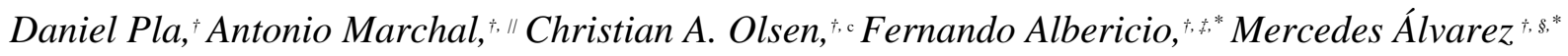

"Biomedical Research Institute, Barcelona Scientific Park-University of Barcelona, 08028 Barcelona; "

Current address: Department of Inorganic and Organic Chemistry, University of Jaén, 23071 Jaén; ' Current address: The Danish University of Pharmaceutical Sciences, Copenhagen, Denmark; ${ }^{\star D}$ Department of Organic Chemistry, University of Barcelona, 08028 Barcelona; ' Laboratory of Organic Chemistry, Faculty of Pharmacy, University of Barcelona, 08028 Barcelona.

\section{TABLE OF CONTENTS}

Experimental Procedures and Characterizations: $\quad$ Page S2-S8

NMR Spectra:

Page S9-S43 


\section{EXPERIMENTAL SECTION}

\section{General}

Tetrahydrofuran was freshly distilled from sodium/benzophenone. Triethylamine was distilled from calcium hydride prior to use and kept in potassium hydroxide. Dimethylsulfoxide was refluxed with calcium hydride, distilled under reduced pressure and kept over $4 \AA$ molecular sieves. Dichloromethane (99.99\% anhydrous) and $N, N$-dimethylformamide (99.99\% anhydrous) were purchased and kept over $4 \AA$ molecular sieves. $N$-Bromosuccinimide was recrystallized previous to use. All organic extracts were dried with anhydrous sodium sufate or magnesium sulfate. Microwave-assisted reactions were carried out in a laboratory microwave oven. The automatic syringe pump was used as specified for controlled addition of some reactants. Automatic flash chromatography was done in a medium pressure liquid cromatograph with commercial silica gel columns (47-60 $\mu \mathrm{m})$. Analytical HPLC samples were run on a $4.6 \times 150 \mathrm{~mm} \mathrm{C}_{18}$ column $(5 \mu \mathrm{m})$ with acetonitrile/water elution and photodiode array detection. Melting points were determined in open capillaries and are uncorrected. ${ }^{1} \mathrm{H}$ NMR and ${ }^{13} \mathrm{C}$ NMR spectra were recorded on a $400 \mathrm{MHz}$ spectrometer. In addition, ${ }^{1} \mathrm{H}$ NMR, and gHSQC were recorded on a $500 \mathrm{MHz}$ spectrometer. Spectra were referenced to appropriate residual solvent peaks $\left(\mathrm{d}_{6}\right.$-acetone, $\mathrm{d}_{6}$-DMSO or $\left.\mathrm{CDCl}_{3}\right)$. IR spectra were obtained on a FT-IR spectrometer. Vibration frequencies are expressed in $\mathrm{cm}^{-1}$. HR-MS were performed on a high resolution mass spectometer by Unidad de Espectrometría de Masas (Universidad de Santiago de Compostela).

The following abbreviations are used: Ac, acetate; CI, chemical ionization; DEAD, diethyl azodicarboxylate; DDQ, 2,3-dichloro-5,6-dicyano-p-benzoquinone; DMAP, 4-dimethylaminopyridine; DMF, $N, N$-dimethylformamide; DPEphos (oxydi-2,1-phenylene)bis(diphenylphosphine); FAB, fast atom bombarding; EI, electronic impact; ESMS, electrospray mass spectrometry; ; gHSQC, gradient heteronuclear single quantum correlation; $m$-CPBA, meta-chloroperoxybenzoic acid; MALDI-TOF, matrix-assisted laser desorption/ionization - time of flight; M.p., melting point; NBS, $N$ bromosuccinimide; NMR, nuclear magnetic resonance; THF, tetrahydrofuran; Ts, $p$-toluenesulfonyl.

\footnotetext{
"Mercedes Álvarez. Tel.: +34 9340370 86; fax: +34 9340371 26; e-mail: malvarez@ pcb.ub.es
} 
3-Isopropoxy-4-methoxyphenol. ${ }^{1}$ A solution of 3-isopropoxy-4-methoxybenzaldehyde (2.68 g, 13.8 mmol) in $\mathrm{CH}_{2} \mathrm{Cl}_{2}(20 \mathrm{~mL})$ was added to a solution of $m$-CPBA (50\% purity, $14.3 \mathrm{~g}, 41.4 \mathrm{mmol}$ ) in $\mathrm{CH}_{2} \mathrm{Cl}_{2}(20 \mathrm{~mL})$, and the mixture was stirred at reflux for $16 \mathrm{~h}$. The solids were then filtered off, and the solvent was removed in vacuo. $\mathrm{NaOH}(1.7 \mathrm{~g})$ in $\mathrm{EtOH} / \mathrm{H}_{2} \mathrm{O}(50: 50,40 \mathrm{~mL})$ was added to the residue, and after stirring for $2 \mathrm{~h}$ the solution was neutralized with $4 \mathrm{~N} \mathrm{HCl}$. The aqueous solution was extracted with $\mathrm{CH}_{2} \mathrm{Cl}_{2}(3 \times 60 \mathrm{~mL})$, and the pooled organic layers were washed with water $(3 \times 75$ $\mathrm{mL}$ ), dried, filtered and concentrated. The residue was purified by flash chromatography on silica gel. Elution with hexane/ $\mathrm{CH}_{2} \mathrm{Cl}_{2}$ (95:5, to 85:15) afforded $1.93 \mathrm{~g}$ (77\%) of a white crystalline solid. M.p. 122.3-122.5 ${ }^{\circ} \mathrm{C}$; IR (film) v 3433, 2977, 1508, $1129 \mathrm{~cm}^{-1} .{ }^{1} \mathrm{H}$ NMR $\left(\mathrm{CDCl}_{3}\right) \delta 1.36(\mathrm{~d}, 6 \mathrm{H}, J=6.4$ $\mathrm{Hz}, 2 \mathrm{Me}$ ); 3.80 (s, 3H, OMe); 4.48 (h, 1H, $J=6.4 \mathrm{~Hz}$ ); 6.34 (dd, 1H, $J=8.8$ and $2.8 \mathrm{~Hz}, \mathrm{H} 6$ ); 6.48 (d, $1 \mathrm{H}, J=2.8 \mathrm{~Hz}, \mathrm{H} 2) ; 6.74(\mathrm{~d}, 1 \mathrm{H}, J=8.8 \mathrm{~Hz}, \mathrm{H} 5) .{ }^{13} \mathrm{C} \mathrm{NMR}\left(\mathrm{CDCl}_{3}\right) \delta 22.0(\mathrm{q}, 2 \mathrm{Me}) ; 56.8(\mathrm{q}, \mathrm{OMe})$; 71.3 (d); 104.2 (d, C2); 106.4 (d, C6); 113.4 (d, C5); 144.6 (s); 148.3 (s); 149.9 (s). MS (CI) 182 (M, 7); $183(\mathrm{M}+1,100) ; 184(\mathrm{M}+2,11)$.

2,4-Diisopropoxy-1-methoxybenzene. ${ }^{2} \quad \mathrm{~K}_{2} \mathrm{CO}_{3}(4.03 \mathrm{~g}, 29.2 \mathrm{mmol})$ and 2-bromopropane (1.9 $\mathrm{mL}$, $20.3 \mathrm{mmol})$ were added to a solution of 3-isopropoxy-4-methoxyphenol (2.65 g, $14.6 \mathrm{mmol})$ in dry DMF $(20 \mathrm{~mL})$. The mixture was microwaved at $80{ }^{\circ} \mathrm{C}$ under Ar for $40 \mathrm{~min}$. The mixture was then cooled to room temperature and the solvent was removed in vacuo. AcOEt was added and the organic solution was washed with $2 \mathrm{M} \mathrm{NaOH}$, saturated $\mathrm{NaHCO}_{3}$, water and brine. The organic layer dried and concentrated gave $2.51 \mathrm{~g}(77 \%)$ of the titled compound as a yellowish oil. IR (film) $\vee 2976,1508$, $1129 \mathrm{~cm}^{-1} .{ }^{1} \mathrm{H}$ NMR $\left(\mathrm{CDCl}_{3}\right) \delta 1.31(\mathrm{~d}, 6 \mathrm{H}, J=6.0 \mathrm{~Hz}, 2 \mathrm{Me}) ; 1.36(\mathrm{~d}, 6 \mathrm{H}, J=6.4 \mathrm{~Hz}, 2 \mathrm{Me}) ; 3.80(\mathrm{~s}$, $3 \mathrm{H}, \mathrm{OMe}) ; 4.42(\mathrm{~h}, 1 \mathrm{H}, J=6.4 \mathrm{~Hz}) ; 4.50(\mathrm{~h}, 1 \mathrm{H}, J=6.0 \mathrm{~Hz}) ; 6.42(\mathrm{dd}, 1 \mathrm{H}, J=8.8$ and $2.8 \mathrm{~Hz}, \mathrm{H} 5)$; $6.52(\mathrm{~d}, 1 \mathrm{H}, J=2.8 \mathrm{~Hz}, \mathrm{H} 3) ; 6.78(\mathrm{~d}, 1 \mathrm{H}, J=8.8 \mathrm{~Hz}, \mathrm{H} 6)$.

1-Bromo-2,4-diisopropoxy-5-methoxybenzene (10). A solution of 2,4-diisopropoxy-1methoxybenzene $(2.51 \mathrm{~g}, 11.2 \mathrm{mmol})$ in dry DMF $(36 \mathrm{~mL})$ was stirred and cooled to $-75^{\circ} \mathrm{C}$. Solid NBS $(1.99 \mathrm{~g}, 11.2 \mathrm{mmol})$ was added, the mixture was stirred for $1 \mathrm{~h}$ at the same temperature and allowed to reach room temperature. The solvent was evaporated and AcOEt was added. The organic solution was filtered through a pad of neutral alumina and concentrated. The residue was purified by flash chromatography on silica gel. Elution with hexane/AcOEt (gradient from 100:0 to 90:10) gave $2.88 \mathrm{~g}$

\footnotetext{
${ }^{1}$ Testaferri, L.; Tiecco, M.; Tingoli, M.; Chianelli, D.; Montanucci, M. Tetrahedron. 1982, 38, 3687.

${ }^{2}$ Iinuma, M.; Iwashima, K.; Matsuura, S. Chemical \& Pharmaceutical Bulletin. 1984, 32, 4935.
} 
$(85 \%)$ of the compound $\mathbf{1 0}$ as a brown oil. IR (film) $\vee 2977,2934,1496,1386,1211,825 \mathrm{~cm}^{-1}$. ${ }^{1} \mathrm{H}$ $\operatorname{NMR}\left(\mathrm{CDCl}_{3}\right) \delta 1.34(\mathrm{~d}, 6 \mathrm{H}, J=6.4 \mathrm{~Hz}, 2 \mathrm{Me}) ; 1.35(\mathrm{~d}, 6 \mathrm{H}, J=6.0 \mathrm{~Hz}, 2 \mathrm{Me}) ; 3.80(\mathrm{~s}, 3 \mathrm{H}, \mathrm{OMe})$; $4.44(\mathrm{~h}, 1 \mathrm{H}, J=6.0 \mathrm{~Hz}) ; 4.46(\mathrm{~h}, 1 \mathrm{H}, J=6.4 \mathrm{~Hz}) ; 6.60(\mathrm{~s}, 1 \mathrm{H}) ; 7.02(\mathrm{~s}, 1 \mathrm{H}) .{ }^{13} \mathrm{C} \mathrm{NMR}\left(\mathrm{CDCl}_{3}\right)$ $\delta 22.2$ (q, 2Me); 22.4 (q, 2Me); 56.8 (q, OMe); 72.4 (d); 74.2 (d) ; 105.4 (s); 108.7 (d); 117.0 (d); 144.4 (s); 147.2 (s); 148.8 (s). MS (CI) $302\left(\mathrm{MBr}^{79}, 92\right) ; 303\left(\mathrm{MBr}^{79}+1,57\right) ; 304\left(\mathrm{MBr}^{81}, 83\right) ; 305$ $\left(\mathrm{MBr}^{81}+1,47\right)$.

2-Bromo-5-isopropoxy-4-methoxystyrene. A solution of methyltriphenylphosphonium bromide (24.6 $\mathrm{g}, 68.9 \mathrm{mmol})$ and DMSO $(3.88 \mathrm{~mL}, 50.3 \mathrm{mmol})$ in freshly distilled THF was stirred and cooled with an ice-bath under Ar and $\mathrm{NaH}(60 \%$ dispersion, $6.04 \mathrm{~g}, 151.0 \mathrm{mmol}$ ) was added. After stirring for additional $30 \mathrm{~min}$ at room temperature 2-bromo-3-isopropoxy-4-methoxybenzaldehyde (13.74 g, 50.3 mmol) was added and the stirring was continued for $30 \mathrm{~min}$. The solvent was removed under reduced pressure and AcOEt $(200 \mathrm{~mL})$ was added. The organic solution was washed with water, $1 \mathrm{~N} \mathrm{HCl}$, saturated $\mathrm{NaHCO}_{3}$, and brine, then dried, filtered and concentrated. The residue was treated with hexane, filtered and the solid was washed with AcOEt. The organic solutions were concentrated and the residue was purified by flash chromatography. Elution with hexane/ $\mathrm{CH}_{2} \mathrm{Cl}_{2}$ (80:20) afforded the bromide, $10.98 \mathrm{~g}(81 \%)$ as a colorless syrup. IR (film) $\vee 1497,1256,1206,1162,912 \mathrm{~cm}^{-1}$. ${ }^{1} \mathrm{H}$ NMR $\left(\mathrm{CDCl}_{3}\right) \delta 1.37(\mathrm{~d}, 6 \mathrm{H}, 3 \mathrm{H}, J=6.1 \mathrm{~Hz}, 2 \mathrm{Me}) ; 3.85(\mathrm{~s}, 3 \mathrm{H}, \mathrm{OMe}) ; 4.55(\mathrm{~h}, 1 \mathrm{H}, J=6.1 \mathrm{~Hz}) ; 5.25(\mathrm{~d}$, $1 \mathrm{H}, J=10.8 \mathrm{~Hz}) ; 5.55(\mathrm{~d}, 1 \mathrm{H}, J=17.2 \mathrm{~Hz}) ; 6.98(\mathrm{dd}, 1 \mathrm{H}, J=17.2$ and $10.8 \mathrm{~Hz}) ; 7.01(\mathrm{~s}, 1 \mathrm{H}) ; 7.09$ (s, 1H). ${ }^{13} \mathrm{C} \mathrm{NMR}\left(\mathrm{CDCl}_{3}\right) \delta 22.0(\mathrm{q}, 2 \mathrm{Me}) ; 56.2$ (q, OMe); 71.9 (d); 113.7 (d); $114.5(\mathrm{t}) ; 114.7(\mathrm{~s})$; 115.8 (d); 129.6 (s); 135.4 (d); 146.7 (s); 151.0 (s). MS (FAB) $270\left(\mathrm{MBr}^{79}, 54\right) ; 271\left(\mathrm{MBr}^{79}+1,26\right)$; $272\left(\mathrm{MBr}^{81}, 57\right) ; 273\left(\mathrm{MBr}^{81}+1,23\right)$.

2-(2-Bromo-5-isopropoxy-4-methoxyphenyl)ethanol. ${ }^{3}$ A solution of 2-bromo-5-isopropoxy-4methoxystyrene $(6.03 \mathrm{~g}, 22 \mathrm{mmol})$ was dissolved in dry THF $(30 \mathrm{~mL})$, cooled on an ice-bath, and $\mathrm{BH}_{3} \bullet \mathrm{SMe}_{2}(12 \mathrm{~mL}, 2.0 \mathrm{M}$ solution in THF, $24.0 \mathrm{mmol})$ was added. The mixture was allowed to reach room temperature and stirred for $3 \mathrm{~h}$. The mixture was cooled to $0{ }^{\circ} \mathrm{C}$, and a solution of $\mathrm{NaOH}(11.27$ g, $282 \mathrm{mmol})$ in EtOH/ $\mathrm{H}_{2} \mathrm{O}(65: 35,30 \mathrm{~mL})$ was added during $1 \mathrm{~h} . \mathrm{H}_{2} \mathrm{O}_{2}(30 \%, 8 \mathrm{~mL}, 70.6 \mathrm{mmol})$ was added and the mixture was stirred at room temperature for $3 \mathrm{~h}$. The solvents were removed under

3 Treu, M.; Jordis, U. Molecules 2002, 7, 374. 
reduced pressure and AcOEt $(200 \mathrm{~mL})$ was added. The organic solution was washed with water and brine, then dried and concentrated. The residue was purified by flash chromatography. Elution with hexane/AcOEt (gradient from 90:10 to 80:20) gave $4.96 \mathrm{~g}$ (77\%) of alcohol as a white solid. M.p. 63.7-65.6 ${ }^{\circ} \mathrm{C}$; IR (film) v 3436, 1600, 1256, 1035, $842 \mathrm{~cm}^{-1} .{ }^{1} \mathrm{H}$ NMR $\left(\mathrm{CDCl}_{3}\right) \delta 1.35(\mathrm{~d}, 6 \mathrm{H}, J=6.4$ $\mathrm{Hz}, 2 \mathrm{Me}), 1.48(\mathrm{br}, 1 \mathrm{H}, \mathrm{OH}) ; 2.93(\mathrm{t}, 2 \mathrm{H}, J=6.8 \mathrm{~Hz}), 3.83(\mathrm{~s}, 3 \mathrm{H}), 3.86$ (t, 2H, J=6.8 Hz), 4.49 (h, $1 \mathrm{H}, J=6.4 \mathrm{~Hz}), 6.82(\mathrm{~s}, 1 \mathrm{H}), 7.03(\mathrm{~s}, 1 \mathrm{H}) .{ }^{13} \mathrm{C} \mathrm{NMR}\left(\mathrm{CDCl}_{3}\right) \delta 22.0(\mathrm{q}, 2 \mathrm{Me}) ; 38.8(\mathrm{t}, \mathrm{C} 2) ; 56.2(\mathrm{q}$, $\mathrm{OMe}$ ); 62.3 (t, C1); 71.8 (d); 116.4 (d); 118.5 (d); 129.6 (s); 146.6 (s); 149.8 (s). MS (CI) 288 $\left(\mathrm{MBr}^{79}\right.$, 26); $289\left(\mathrm{MBr}^{79}+1,11\right) ; 290\left(\mathrm{MBr}^{81}, 27\right) ; 291\left(\mathrm{MBr}^{81}+1,7\right)$. HRMS m/z calcd for $\mathrm{C}_{12} \mathrm{H}_{18} \mathrm{O}_{3} \mathrm{Br}^{+} 289.0439$, found 289.0430.

2-(2-Bromo-5-isopropoxy-4-methoxyphenyl)ethyl p-toluenesulfonate (4). A solution of TsCl (11.98 $\mathrm{g}, 62.25 \mathrm{mmol})$ in $\mathrm{CH}_{2} \mathrm{Cl}_{2}(100 \mathrm{~mL})$ was added to a cooled solution of 2-(2-bromo-5-isopropoxy-4methoxyphenyl)ethanol (15.0 g, $51.87 \mathrm{mmol}), \mathrm{Et}_{3} \mathrm{~N}(7.94 \mathrm{~mL}, 57.06 \mathrm{mmol})$ and DMAP (63.5 mg, 0.51 mmol) in $\mathrm{CH}_{2} \mathrm{Cl}_{2}(500 \mathrm{~mL})$. The reaction mixture was allowed to reach room temperature and was stirred for $8 \mathrm{~h}$. The solution was washed with water, $2 \mathrm{~N} \mathrm{HCl}$, saturated $\mathrm{NaHCO}_{3}$ and brine, then dried and concentrated. Hexane was added to the residue to yield 4 (19.31 g, $84 \%)$ as a white solid isolated by filtration. IR (film) $\vee 1361 ; 1176 ; 1188 \mathrm{~cm}^{-1}$. ${ }^{1} \mathrm{H}$ NMR $\left(\mathrm{CDCl}_{3}\right) \delta 1.35(\mathrm{~d}, 6 \mathrm{H}, J=6.0 \mathrm{~Hz}, 2 \mathrm{Me})$; $2.43(\mathrm{~s}, 3 \mathrm{H}, \mathrm{Me}) ; 2.99$ (t, 2H, J= $7.2 \mathrm{~Hz}) ; 3.81$ (s, 3H, OMe); 4.21 (t, 2H, J=7.2 Hz); 4.45 (h, 1H, $J=$ $6.0 \mathrm{~Hz}) ; 6.72(\mathrm{~s}, 1 \mathrm{H}) ; 6.92(\mathrm{~s}, 1 \mathrm{H}) ; 7.28(\mathrm{~d}, 2 \mathrm{H}, J=6.4 \mathrm{~Hz}) ; 7.69(\mathrm{~d}, 2 \mathrm{H}, J=6.4 \mathrm{~Hz}) .{ }^{13} \mathrm{C}$ NMR $\left(\mathrm{CDCl}_{3}\right) \delta 21.6(\mathrm{q}) ; 22.0(\mathrm{q}, 2 \mathrm{Me}) ; 35.3(\mathrm{t}, \mathrm{C} 2) ; 56.2$ (q, OMe); 69.1 (t, C1); 71.8 (d); $114.5(\mathrm{~s}) ; 116.1$ (d); $118.4(\mathrm{~d}) ; 127.3(\mathrm{~s}) ; 127.8(\mathrm{~d}, 2 \mathrm{CH}) ; 129.7$ (d, 2CH); 133.0 (s); 144.6 (s); 146.6 (s); 150.1 (s). MS (CI) $442\left(\mathrm{MBr}^{79}, 38\right) ; 443\left(\mathrm{MBr}^{79}+1,14\right) ; 444\left(\mathrm{MBr}^{81}, 36\right) ; 445\left(\mathrm{MBr}^{81}+1,10\right)$.

Methyl 1-[2-bromo-5-isopropoxy-4-methoxyphenethyl]-1H-pyrrole-2-carboxylate. Methyl 1H-pyrrole2-carboxylate $(3.38 \mathrm{~g}, 27.0 \mathrm{mmol})$ and $\mathrm{NaH}(60 \%$ dispersion, $988 \mathrm{mg}, 24.7 \mathrm{mmol})$ were stirred in dry DMF (25 mL) on an ice-bath for $30 \mathrm{~min}$. A solution of $4(5.98 \mathrm{~g}, 13.5 \mathrm{mmol})$ in dry DMF $(35 \mathrm{~mL}) \mathrm{was}$ added and the mixture was stirred for $5 \mathrm{~h}$ at room temperature. The DMF was then evaporated under reduced pressure, and $\mathrm{Et}_{2} \mathrm{O}$ was added. The organic solution was washed with $1 \mathrm{~N} \mathrm{HCl}$, saturated $\mathrm{NaHCO}_{3}$ and brine, then dried and concentrated. The residue was purified by flash chromatography. Elution with hexane/AcOEt (gradient from 95:5 to 90:10) gave $4.34 \mathrm{~g}$ (81\%) of the title compound as a white solid. M.p. $63{ }^{\circ} \mathrm{C}$. IR (film) v 1706, 1497, 1244, 1107, $739 \mathrm{~cm}^{-1}$. ${ }^{1} \mathrm{H}$ NMR $\left(\mathrm{CDCl}_{3}\right) \delta 1.27$ $(\mathrm{d}, 6 \mathrm{H}, J=6.0 \mathrm{~Hz}, 2 \mathrm{Me}) ; 3.08(\mathrm{t}, 2 \mathrm{H}, J=6.8 \mathrm{~Hz}) ; 3.80$ (s, 3H, OMe); 3.82 (s, 3H, OMe); $4.34(\mathrm{~h}, 1 \mathrm{H}$, $J=6.0 \mathrm{~Hz}, \mathrm{CH}) ; 4.50(\mathrm{t}, 2 \mathrm{H}, J=6.8 \mathrm{~Hz}) ; 5.99(\mathrm{dd}, 1 \mathrm{H}, J=4.0$ and $2.0 \mathrm{~Hz}, \mathrm{H} 3) ; 6.46(\mathrm{~s}, 1 \mathrm{H}) ; 6.55(\mathrm{t}$, 
$1 \mathrm{H}, J=2.0 \mathrm{~Hz}, \mathrm{H} 5) ; 6.93(\mathrm{dd}, 1 \mathrm{H}, J=4.0$ and $2.0 \mathrm{~Hz}, \mathrm{H} 4) ; 7.00(\mathrm{~s}, 1 \mathrm{H}) .{ }^{13} \mathrm{NMR}\left(\mathrm{CDCl}_{3}\right) \delta 22.0(\mathrm{q}$, 2Me); 37.7 (t); 48.9 (t); 51.0 (q); 56.2 (q); 71.7 (d); 107.8 (d); 114.5 (s); 116.0 (d); 118.2 (d); 118.4 (d); 121.3 (s); 129.2 (d); 129.6 (s); 146.6 (s); 149.8 (s); 161.5 (s, C=O). MS (EI) 395 (MBr $\left.{ }^{79}, 1\right) ; 397$ $\left(\mathrm{MBr}^{81}, 1\right)$. HRMS $m / z$ calcd for $\mathrm{C}_{18} \mathrm{H}_{22} \mathrm{NO}_{4} \mathrm{Br} 395.0732$, found 395.0723.

Methyl 8-isopropoxy-9-methoxy-5,6-dihydropyrrolo[2,1-a]isoquinoline-3-carboxylate (1). A mixture of methyl 1-[2-bromo-5-isopropoxy-4-methoxyphenethyl]-1H-pyrrole-2-carboxylate (307 mg, 0.78 $\mathrm{mmol})$ in dry $\mathrm{DMF}(5 \mathrm{~mL}), \mathrm{K}_{2} \mathrm{CO}_{3}(235 \mathrm{mg}, 1.71 \mathrm{mmol}), \mathrm{PPh}_{3}(163 \mathrm{mg}, 0.62 \mathrm{mmol})$, and $\mathrm{PdCl}_{2}\left(\mathrm{PPh}_{3}\right)_{2}(218 \mathrm{mg}, 0.31 \mathrm{mmol})$ was purged with Ar for $15 \mathrm{~min}$. The reaction mixture was stirred for $20 \mathrm{~h}$ at $125^{\circ} \mathrm{C}$. After cooling to room temperature, the DMF was removed in vacuo, and $\mathrm{Et}_{2} \mathrm{O}(100$ $\mathrm{mL}$ ) was added. The organic solution was washed with water and brine, dried, filtered through a pad of celite and concentrated. The residue was purified by flash chromatography. Elution with hexane/AcOEt (gradient from 95:5 to 85:15) gave 1 (199 mg, 81\%) as a white solid. M.p. $109{ }^{\circ} \mathrm{C}$. IR $\left(\right.$ film) $\vee 1699,1489,1434,1248,1130 \mathrm{~cm}^{-1}$. ${ }^{1} \mathrm{H}$ NMR $\left(\mathrm{CDCl}_{3}\right) \delta 1.39(\mathrm{~d}, 6 \mathrm{H}, J=6.0 \mathrm{~Hz}, 2 \mathrm{Me}) ; 2.98$ $(\mathrm{t}, 2 \mathrm{H}, J=6.8 \mathrm{~Hz}, \mathrm{H6}) ; 3.82(\mathrm{~s}, 3 \mathrm{H}, \mathrm{OMe}) ; 3,88(\mathrm{~s}, 3 \mathrm{H}, \mathrm{OMe}) ; 4.56(\mathrm{~h}, 1 \mathrm{H}, J=6.0 \mathrm{~Hz}, \mathrm{OCH}) ; 4.60(\mathrm{t}$, $2 \mathrm{H}, J=6.8 \mathrm{~Hz}, \mathrm{H} 5) ; 6.41(\mathrm{~d}, 1 \mathrm{H}, J=4.0 \mathrm{~Hz}, \mathrm{H} 1) ; 6.75$ (s, 1H, H7); 7.00 (d, 1H, J= 4.0 Hz, H2); 7.05 $(\mathrm{s}, 1 \mathrm{H}, \mathrm{H} 10) .{ }^{13} \mathrm{C} \mathrm{NMR}\left(\mathrm{CDCl}_{3}\right) \delta 22.1(\mathrm{q}) ; 28.4(\mathrm{t}) ; 42.2(\mathrm{t}) ; 50.9(\mathrm{q}) ; 56.2(\mathrm{q}) ; 71.6(\mathrm{~d}) ; 103.4(\mathrm{~d})$; 107.7 (d); 115.3 (d); 118.4 (d); 121.2 (s); 121.3 (s); 124.5 (s); 136.4 (s); 147.0 (s); 149.6 (s); 161.7 (s, $\mathrm{C}=\mathrm{O})$. $\quad \mathrm{MS}(\mathrm{EI}) 315(\mathrm{M}, 56) ; 316(\mathrm{M}+1,12) ; 317(\mathrm{M}+2,4)$. HRMS m/z calcd for $\mathrm{C}_{18} \mathrm{H}_{21} \mathrm{NO}_{4}$ 315.1471, found 315.1476 .

Methyl 1-bromo-8-isopropoxy-9-methoxy-5,6-dihydropyrrolo[2,1-a]isoquinoline-3-carboxylate (5). A mixture of 1 (1.46 g, $4.64 \mathrm{mmol})$ and NBS (835 mg, $4.69 \mathrm{mmol})$ dissolved in THF (40 mL) was stirred at $70{ }^{\circ} \mathrm{C}$ under Ar for $3 \mathrm{~h}$. After cooling to room temperature the solvent was removed in vacuo, and the resulting residue was purified by column chromatography on silica gel. Elution with hexane/AcOEt (95:5 and 90:10) afforded 5 (1.72 g, 94\%) as white crystals. M.p. 113.5-119.4 ${ }^{\circ} \mathrm{C}$. IR (film) 1705, 1440, 1245, 1213, 1197, 1066, $758 \mathrm{~cm}^{-1}$. ${ }^{1} \mathrm{H}$ NMR $\left(\mathrm{CDCl}_{3}\right) \delta 1.41$ (d, 6H, J = 6.0, 2Me); $2.94(\mathrm{~d}, 2 \mathrm{H}, J=6.8 \mathrm{~Hz}, \mathrm{H} 6) ; 3.81$ (s, 3H, OMe); 3.91 (s, 3H, OMe); 4.61-4.54 (m, 3H, H5 and OCH); 6.76 (s, 1H, H2); 7.01 (s, 1H, H7); 7.97 (s, 1H, H10). ${ }^{13} \mathrm{C} \mathrm{NMR}\left(\mathrm{CDCl}_{3}\right) \delta 22.1$ (q); $29.0(\mathrm{t}) ; 42.6(\mathrm{t})$; 51.2 (q); 56.1 (q); 71.5 (d); 92.5 (s); 108.8 (d); 114.9 (d); 120.4 (s); 120.5 (s); 121.0 (d); 125.7 (s); 131.5 (s); 147.0 (s); 149.0 (s); 160.9 (s, C=O). MS (CI) $393\left(\mathrm{MBr}^{79}, 84\right) ; 394\left(\mathrm{MBr}^{79}+1,100\right) ; 395$ $\left(\mathrm{MBr}^{81}, 82\right) ; 396\left(\mathrm{MBr}^{81}+1,89\right)$. HRMS $m / z$ calcd for $\mathrm{C}_{18} \mathrm{H}_{21} \mathrm{NO}_{4} \mathrm{Br}^{+}$395.0732, found 394.0645. 
Methyl 1-(4-hydroxy-3-methoxyphenyl)-8-isopropoxy-9-methoxy-5,6-dihydropyrrolo[2,1-a]isoquinoline-3-carboxylate (7). A mixture of 5 (249 $\mathrm{mg}, 0.63 \mathrm{mmol}), 6(237 \mathrm{mg}, 0.95 \mathrm{mmol})$, and $2 \mathrm{M} \mathrm{Na}_{2} \mathrm{CO}_{3}$ $(1.26 \mathrm{~mL})$ in DMF $(10 \mathrm{~mL})$ was purged with Ar for 10 minutes. Then $\operatorname{Pd}\left(\mathrm{PPh}_{3}\right)_{4}(147 \mathrm{mg}, 0.126$ mmol) was added and Ar was bubbled through the mixture for 5 minutes. The temperature was raised to $125^{\circ} \mathrm{C}$ and stirring was continued for $16 \mathrm{~h}$. After cooling, the solvents were evaporated in vacuo, and $\mathrm{Et}_{2} \mathrm{O}$ was added. The organic solution was washed with water and brine, dried, and concentrated. The residue was purified by flash chromatography. Elution with hexane/AcOEt (50:50 and 40:60) gave 7 (215 mg, 78\%) as a light brown solid. M.p. 176-180.9 ${ }^{\circ} \mathrm{C}$; IR (film) v 3435, 3023, 1698, 1464, 14401239, 1195, $760 \mathrm{~cm}^{-1} .{ }^{1} \mathrm{H}$ NMR $\left(\mathrm{CDCl}_{3}\right) \delta 1.37(\mathrm{~d}, 6 \mathrm{H}, J=6.0 \mathrm{~Hz}, 2 \mathrm{Me}) ; 2.99(\mathrm{t}, 2 \mathrm{H}, J=6.4 \mathrm{~Hz}$, H6); 3.42 (s, 3H, OMe); 3.84 (s, 6H, 2OMe); 4.53 (h, 1H, $J=6.0 \mathrm{~Hz}, \mathrm{CH}$ ); 4.60 (t, 2H, $J=6.4 \mathrm{~Hz}$, H5); 5.62 (br, 1H, OH); 6.74 (s, 1H, H2); 6.89 (s, 1H, H7); 6.92 (d, 1H, J = $8.0 \mathrm{~Hz}, \mathrm{H} 5$ '); 6.94 (d, 1H, $J=2.0 \mathrm{~Hz}, \mathrm{H} 2$ '); 6.96 (dd, $1 \mathrm{H}, J=8.0$ and $2.0 \mathrm{~Hz}, \mathrm{H} 6$ '); 6.97 (s, $1 \mathrm{H}, \mathrm{H} 10) .{ }^{13} \mathrm{C} \mathrm{NMR}\left(\mathrm{CDCl}_{3}\right) \delta 22.1$ (q, Me); 29.0 (t, C6); 42.5 (t, C5); 51.1 (q, Me); 55.5 (q, Me); 56.0 (q, Me); 71.4 (d, OCH); 109.2 (d,C7); 112.0 (d, C5'); 114.3 (d, C6'); 114.7 (d, C2); 119.2 (d, C10); 120.0 (s); 121.2 (s); 121.7 (s); 122.4 (d, C2); 125.7 (s); 128.6 (s); 131.7 (s); 144.5 (s); 146.4 (s); 146.5 (s); 148.6 (s); 161.8 (s). MS (EI) 437 (M, 92); $438(\mathrm{M}+1,47) ; 396$ (56); 395 (100); 394 (47). HRMS m/z calcd for $\mathrm{C}_{25} \mathrm{H}_{27} \mathrm{NO}_{6}$ 437.1838, found 437.1833 .

Methyl 8-isopropoxy-1-(4-isopropoxy-3-methoxyphenyl)-9-methoxy-5,6-dihydropyrrolo[2,1-a]isoquinoline-3-carboxylate $(\mathbf{8}) . \mathrm{K}_{2} \mathrm{CO}_{3}(271 \mathrm{mg}, 1.96 \mathrm{mmol})$ and 2-bromopropane $(1.2 \mathrm{~mL}, 12.83 \mathrm{mmol})$ were added to 7 (419 mg, $0.96 \mathrm{mmol}$ ) in dry DMF (6 mL). The mixture was stirred at $80^{\circ} \mathrm{C}$ under $\mathrm{Ar}$ for $17 \mathrm{~h}$. The mixture was then cooled to room temperature and the solvent removed in vacuo. AcOEt was added and the organic solution was washed with water, saturated $\mathrm{NaHCO}_{3}$ and brine. The organic layer was then dried, filtered and concentrated. The residue was purified by flash chromatography. Elution with hexane/AcOEt (50:50) gave 8 (379 mg, 83\%) as a light brown solid. IR (film) v 1700, 1464, 1440, 1423, $1192 \mathrm{~cm}^{-1}$. ${ }^{1} \mathrm{H}$ NMR $\left(\mathrm{CDCl}_{3}\right) \delta 1.37(2 \mathrm{~d}, 12 \mathrm{H}, J=6.0 \mathrm{~Hz}, 4 \mathrm{Me}) ; 3.00(\mathrm{t}, 2 \mathrm{H}, J=$ $6.8 \mathrm{~Hz}, \mathrm{H6}$ ); 3.40 (s, 3H, OMe); 3.81 (s, 3H, OMe); 3.84 (s, 3H, OMe); 4.53 (h, 2H, J=6.0 Hz, 2CH); 4.61 (t, 2H, $J=6.8$ Hz, H5); 6.74 (s, 1H, H2); 6.90 (s, 1H, H7); 6.92 (br, 1H, H2'); 6.94-6.98 (m, 2H, H6' and H5'); 6.99 (s, 1H, H10). ${ }^{13} \mathrm{C} \mathrm{NMR}_{\left(\mathrm{CDCl}_{3}\right)} \delta 22.1$ (2q, 4Me); 29.0 (t, C6); 42.6 (t, C5); 51.1 (q, OMe); 55.4 (q, OMe); 56.0 (q, OMe); 71.4 (d, OCH); 71.6 (d, OCH); 109.2 (d, C7); 113.3 (d, C2'); 114.7 (d, C2); 116.4 (d, C5'); 119.3 (d, C10); 120.1 (s); 121.2 (s); 121.7 (d, C6'); 125.7 (s); 129.8 (s); 131.7 (s); 146.1 (s); 146.5 (s); 148.6 (s); 150.4 (s); 161.8 (s, C=O). MS (CI) 479 (M, 78); $480(\mathrm{M}+1,100) ; 481(\mathrm{M}+2,40)$. HRMS $m / z$ calcd for $\mathrm{C}_{28} \mathrm{H}_{33} \mathrm{NO}_{6} 479.2308$, found 479.2320. 
Methyl 2-bromo-8-isopropoxy-1-(4-isopropoxy-3-methoxyphenyl)-9-methoxy-5,6-dihydropyrrolo[2,1a isoquinoline-3-carboxylate (9). Compound 8 (756 mg, $1.58 \mathrm{mmol})$ was dissolved in freshly distilled THF, and NBS (339 mg, $1.90 \mathrm{mmol}$ ) was added in one portion. The mixture was stirred under Ar for $1.5 \mathrm{~h}$ at $70{ }^{\circ} \mathrm{C}$. The solvent was removed under reduced pressure and the residue was purified by flash chromatography. Elution with hexane/AcOEt (gradient from 75:25 to 50:50) gave 9 (741 mg, 84\%) as a yellow solid. IR (film) $\vee 1694,1463,1440,1111 .{ }^{1} \mathrm{H}$ NMR $\left(\mathrm{CDCl}_{3}\right) \delta 1.35(\mathrm{~d}, 6 \mathrm{H}, J=8.0 \mathrm{~Hz}$, 2Me); 1.38 (d, 6H, $J=8.0 \mathrm{~Hz}, 2 \mathrm{Me}) ; 2.98$ (t, 2H, $J=6.0 \mathrm{~Hz}, \mathrm{H6}$ ); 3.30 (s, 3H, OMe); 3.81 (s, 3H, OMe); 3.90 (s, 3H, OMe); 4.54 (m, 4H, H5 and 2OCH); 6.57 (s, 1H, H7); 6.70 (s, 1H, H10); 6.87 (dd, $1 \mathrm{H}, J=7.9$ and $2.0 \mathrm{~Hz}, \mathrm{H6}$ '); 6.88 (br, $1 \mathrm{H}, \mathrm{H} 2$ '); 6.96 (d, $\left.J=7.9 \mathrm{~Hz}, 1 \mathrm{H}, \mathrm{H} 5^{\prime}\right) .{ }^{13} \mathrm{C}^{\mathrm{NMR}}\left(\mathrm{CDCl}_{3}\right) \delta$ 22.1 (q, 4Me); 28.7 (t, C6); 43.5 (t, C5); 51.2 (q, OMe); 55.1 (q, OMe); 56.1 (q, OMe); 71.4 (d, 2H, OCH); 107.7 (s, C2); 109.1 (d, C7); 114.6 (d, C10); 114.8 (d, C6'); 115.7 (d, C5'); 118.5 (s); 120.2 (s, C10a); 122.6 (s, C1'); 123.4 (s, C1); 125.5 (s, C6a); 127.6 (s, C4'); 132.1 (s, C10b); 146.8 (s, C8); 148.6 (s, C9); 150.4 (s, C3'); 161.4 (s, C=O). MS (CI) $560\left(\mathrm{MBr}^{81}, 84\right) ; 559\left(\mathrm{MBr}^{79}+1\right) ; 558\left(\mathrm{MBr}^{79}\right)$. HRMS $m / z$ calcd for $\mathrm{C}_{28} \mathrm{H}_{32} \mathrm{NO}_{6} \mathrm{Br} 557.1413$, found 557.1429.

2-(2,4-Diisopropoxy-5-methoxyphenyl)-boronic acid (11a). $n \mathrm{BuLi}(10.3 \mathrm{~mL}, 0.4 \mathrm{M}$ in hexane, 4.12 mmol) was added to 1-bromo-2,5-diisopropoxy-4-methoxybenzene (832 $\mathrm{mg}, 2.74 \mathrm{mmol})$ in dry THF $(20 \mathrm{~mL})$ at $-78^{\circ} \mathrm{C}$ under $\mathrm{Ar}$ and was stirred for $15 \mathrm{~min}$. Triisopropyl borate $(0.95 \mathrm{~mL}, 4.12 \mathrm{mmol})$ was added and the mixture was allowed to reach room temperature and stirred overnight. The solvent was evaporated under reduced pressure and the resulting crude was quenched with water at $0{ }^{\circ} \mathrm{C}$ and $2 \mathrm{M}$ $\mathrm{HCl}$. The aqueous solution was extracted with $\mathrm{Et}_{2} \mathrm{O}$. The organic solution dried and concentrated afforded a crude material which was purified by flash column chromatography. Elution with hexane/AcOEt (gradient from 85:15 to 80:20) gave $147 \mathrm{mg}$ (20\%) of 11a as a brown syrup. ${ }^{1} \mathrm{H}$ NMR $\left(\mathrm{CDCl}_{3}, 400 \mathrm{MHz}\right) \delta 1.38(\mathrm{~d}, 6 \mathrm{H}, J=6.0 \mathrm{~Hz}, 2 \mathrm{Me}) ; 1.39$ (d, 6H, $\left.J=6.4 \mathrm{~Hz}, 2 \mathrm{Me}\right) ; 3.85(\mathrm{~s}, 3 \mathrm{H}, \mathrm{OMe})$; 4.55-4.58 (m, 2H); $5.81(\mathrm{~s}, 1 \mathrm{H}) ; 6.53(\mathrm{~s}, 1 \mathrm{H})$. 